\title{
Spatially-Resolved View of High-Redshift Starbursts: the case of Sub-mm Galaxies
}

\author{
Karín Menéndez-Delmestre ${ }^{1}$, Andrew W. Blain ${ }^{2}$, Mark Swinbank $^{3}$, \\ Ian Smail ${ }^{3}$, Rob J. Ivison ${ }^{4,5}$, and Scott C. Chapman ${ }^{6}$ \\ ${ }^{1}$ Observatório do Valongo, Universidade Federal do Rio de Janeiro, Ladeira do Pedro Antônio \\ 43, Rio de Janeiro, RJ 20080-090, Brazil; email: kmd@astro.ufrj.br \\ ${ }^{2}$ University of Leicester, University Road, Leicester, LE1 7RH, UK \\ ${ }^{3}$ Institute for Computational Cosmology, Durham University, Durham DH1 3LE, UK \\ ${ }^{4}$ UK Astronomy Technology Centre, Blackford Hill, Edinburgh EH9 3HJ \\ ${ }^{5}$ Institute for Astronomy, Blackford Hill, Edinburgh EH9 3HJ \\ ${ }^{6}$ Institute of Astronomy, Madingley Road, Cambridge, CB3 0HA, UK
}

\begin{abstract}
Ultra-luminous infrared galaxies $\left(\mathrm{L}_{I R}>10^{12} \mathrm{~L}_{\odot}\right)$ are locally rare, but appear to dominate the co-moving energy density at higher redshifts $(z>2)$. Many of these are opticallyfaint, dust-obscured galaxies that have been identified by the detection of their thermal dust emission at sub-mm wavelengths. Multi-wavelength spectroscopic follow-up observations of these sub-mm galaxies $(\mathrm{SMGs})$ have shown that they are massive $\left(\mathrm{M}_{\text {stellar }} \sim 10^{11} \mathrm{M}_{\odot}\right)$ objects undergoing intense star-formation (SFRs $\sim 10^{2}-10^{3} \mathrm{M}_{\odot} \mathrm{yr}^{-1}$ ) with a mean redshift of $z \sim 2$, coinciding with the epoch of peak quasar activity. The large fraction of AGNs in SMGs and the derived SMBH masses $\left(\mathrm{M}_{\bullet}<10^{8} \mathrm{M}_{\odot}\right)$ in these galaxies suggest that the submm phase may play an important role in the rapid growth of SMBHs. When both AGN and star-formation activity are present, long-slit spectroscopic techniques face difficulties in disentangling their contributions and may result in SFR and mass overestimates. We present an integral field view of the $\mathrm{H} \alpha$ emission in a sample of 3 SMGs at $z \sim 1.4-2.4$ with the IFU instrument OSIRIS on Keck. Designed to be used with Laser Guide Star Adaptive Optics, OSIRIS allows a spatial resolution of up to $10 \times$ higher than what has been possible in previous seeing-limited studies of the ionized gas in these galaxies. Our main results are the following: (1) We detect multiple galactic-scale sub-components: the compact, broad $\mathrm{H} \alpha$ emission (FWHM $>1000 \mathrm{~km} \mathrm{~s}^{-1}$ ) likely associated with an AGN, the more extended narrow-line $\mathrm{H} \alpha$ emission (FWHM $\lesssim 500 \mathrm{~km} \mathrm{~s}^{-1}$ ) of star-forming regions; the latter are dominated by multiple $1-2 \mathrm{kpc}$ sized $\mathrm{H} \alpha$-bright clumps, each contributing $1-25 \%$ of the total clump-integrated $\mathrm{H} \alpha$ emission. (2) We derive clump dynamical masses $\sim 1-10 \times 10^{9} \mathrm{M}_{\odot}, 1-2$ orders of magnitude larger than the kpc-scaled stellar clumps uncovered in optically-selected $z \sim 2$ star-forming galaxies. (3) We determine high star-formation rate surface densities $\left(\Sigma_{\mathrm{SFR}} \sim 1-50 \mathrm{M}_{\odot} \mathrm{yr}^{-1} \mathrm{kpc}^{-2}\right.$, after extinction correction), similar to local starbursts and luminous infrared galaxies. In contrast to these local environments, SMGs undergo such intense activity on significantly larger spatial scales as revealed by extended $\mathrm{H} \alpha$ emission over 4-16 kpc. (4) We find no evidence of ordered global motion as it would be found in a disk, but rather large velocity offsets $\left(\sim\right.$ few $\left.\times 100 \mathrm{~km} \mathrm{~s}^{-1}\right)$ between the distinct stellar clumps. The merger interpretation is likely the most accurate scenario for the SMGs in our sample. However, the final test of whether an underlying disk structure is present will come from studies of the cold gas at the high spatial resolutions possible with ALMA.
\end{abstract}

We refer the reader to Menéndez-Delmestre et al. (2012) for more details.

Keywords. galaxies: high-redshift, galaxies: kinematics and dynamics, galaxies: starburst, galaxies: active, infrared: galaxies, submillimeter, techniques: spectroscopic, instrumentation: adaptive optics 